\title{
ANALYSIS OF MODIFIED DISCRETIZATION METHODS FOR THE MEASUREMENT VOLUME
}

\author{
D. Mariūnas, V. Giniotis
}

Vilnius Gediminas Technical University.E-mail: Darius.mariunas@ai.vtu.lt Received 2307 2004, accepted 26072004

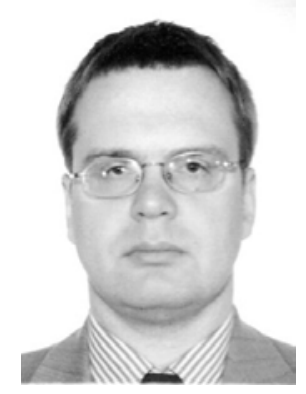

Darius MARIŪNAS was born in Vilnius, Lithuania on 24 September 1971. 1994, VGTU, received a Diploma of Mechanical Engineering; 1995, VGTU, master's degree in Mechanical Engineering. From 2000 - doctoral studies in Measurement Engineering, 10T, VGTU. Field of scientific research - measurement information, performance of measurements by discretization of measuring space. Publications: five research reports and seven papers.

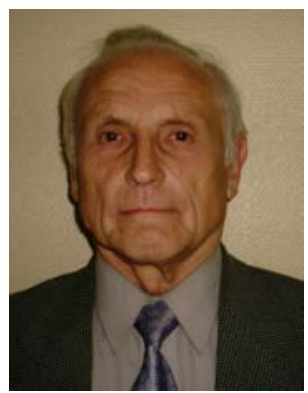

Vytautas GINIOTIS, Prof Dr Habil

Affiliations and functions: Engineer at various machine production factories; 1989 - 1994 - Chief metrologist at Experimental Scientific Institute of Metal Cutting Machines (ENIMS) and in Vilnius State Grinding Machines factory; 1994 - 2003 - Head of the Department of Aviation Technologies at Antanas Gustaitis Aviation Institute of Vilnius Gediminas Technical University

Experience: Have made presentations and taken part in many international scientific conferences. Have visited Universities in England, Sweden, Italy, Austria. Inventions: 50 inventions. Publications. Author more than 150 scientific articles, including 3 booklets.

Present position: Chief Scientific Worker at Institute of Geodesy, VGTU.

\begin{abstract}
The analysis performed in the paper shows that the effectiveness of discretization methods depends on the accuracy of the evaluation of the parameters of local surface errors and on the characteristics of the regression polynomial describing them. It is evident from the expressions derived that the wavelength of the distribution errors depends on the number of members of the regression polynomial. By increasing the number of members of the regression polynomial, the wavelength of errors of the surface form will be not evaluated. On the other hand, reducing the number of polynomial members, the accuracy of the description of local surface errors will be lost. This is why a priori information is needed about the surface to be measured before choosing the order of the polynomial equation.
\end{abstract}

Keywords: CMM, measurement, volume discretization, errors, method analysis.

\section{Introduction}

Two main tasks must be fulfilled during coordinate measurements: to select the information about the errors of the object and to reduce to a minimum the number of points to be measured. This is more important when using coordinate measuring machines $(\mathrm{CMM})$ or during the calibration of the CMM itself for their error determination in the multidimensional ( $\mathrm{n}$ - dimensional) volume. Various measurement models and methods are used for sampling strategy during the selection of the optimal number of points in the measuring volume. It is widely analyzed in several works of research $[4,5,9$, 10], etc. Successions models as Hamersley, Halton - Zaremba are used in which the coordinates of measurement points are calculated according to the particular formulas for the flatness measurement [10, 5]. Kim and Rumant use the systematic straight and systematic random models for set of measurement points that are predicated on various successions [4]. Yau and Meng [11] propose a hierarchy- planned system for the determination errors using CMM. This allows selecting the measurement process and the coordinates for the measurement of errors to be selected [11]. Some authors use the "gray" theory to prognosticate measurement points, B - splines and other models of parameter modeling [6, 1]. Analyzing three-dimensional space and the law of the distribution of flatness error, various mathematical models then are used, which allow the value of errors in the measuring space or plane to be evaluated [9, 11, 1]. The most analyzed methods are associated with the set of mathematical models, generated series or splines. The coordinates of the measurement errors often depend on the type of spline, and if it is be changed, then another set of coordinates will be generated. Besides, the coordinates for error measurement particularly depend on the model chosen for splitting the space or the plain into pitches (steps) for measurements to be performed. The area, where errors vary evenly, the pitch of discretization can be chosen larger and that, where variation is quite sharp - significantly less. The 
methods discussed above are connected with mathematical models based on sequences or the generation of splines. The coordinates determined in such way a depend on the type of spine, and they will be different if the spline is changed. The measurement strategy is strongly dependant on the method of splitting the area or volume that is to be measured.

The papers [8, 7 and 3] present a modified volume discretization method where the pitch of the discretization is calculated in inverse ratio to the values of the gradient module or its constituents. Nevertheless, there is no analysis made of the possibilities of their antecedent application. There is only mentioned that their effectiveness is less when the wavelength of the error's pitch is small. The purpose of this work is to investigate the methods proposed in papers $[8,7]$ and to determine the wavelength of constituents of the errors to which the methods proposed are not quite effective.

The purpose of this work is the analysis of the possibilities of discretization of two- and threedimensional space, evaluating the trend of the function of the systematic error gradient in the $\mathrm{x}, \mathrm{y}$, and $\mathrm{z}$ axe; selection the trends for the new measurement plan, to determine relation of the dimensions to the mean differential parameters of the gradient. By splitting the measurement volume in such manner, measurement coordinates will only depend on the characteristics of systematic errors. An implementation of this method in the measurement strategy is also discussed.

\section{Object and methods}

A modified volume discretization method is proposed in papers 8,7 and 3 where the pitch of the discretization is calculated in inverse ratio to the values:

- of the gradient module:

$$
\begin{aligned}
& L_{\mathrm{int}}=q \frac{L}{|\bar{\Delta}|}, \\
& |\stackrel{\mathrm{s}}{\Delta}|=\sqrt{\left(\frac{\partial \Phi}{\partial x}\right)^{2}+\left(\frac{\partial \Phi}{\partial y}\right)^{2}+\left(\frac{\partial \Phi}{\partial z}\right)^{2}} ;
\end{aligned}
$$

- of the constituents of the gradient module:

$$
L_{\text {int } x}=q \cdot \frac{L}{\left|\frac{\partial \Phi}{\partial x}\right|}, \quad L_{\text {int } y}=q \cdot \frac{L}{\left|\frac{\partial \Phi}{\partial y}\right|}, \quad L_{\text {int } z}=q \cdot \frac{L}{\left|\frac{\partial \Phi}{\partial z}\right|}
$$

Here $\Phi$ is an analytic expression of the local errors on the surface; $O x, O y, O z$ are coordinate axes; $L$ is the value of the pitch of discretization of the surface by standard methods; and $q$ is coefficient of proportion.

Having in mind that the value of partial derivatives is $\operatorname{tg} \alpha$, then it is valid to write: $\frac{\partial \Phi}{\partial x}=\operatorname{tg} \alpha_{x} ; \frac{\partial \Phi}{\partial y}=\operatorname{tg} \alpha_{y} ; \quad \frac{\partial \Phi}{\partial z}=\operatorname{tg} \alpha_{z}$, where the value of $\left.\right|^{\prime} \Delta \mid$ will be equal to some special calculated value of

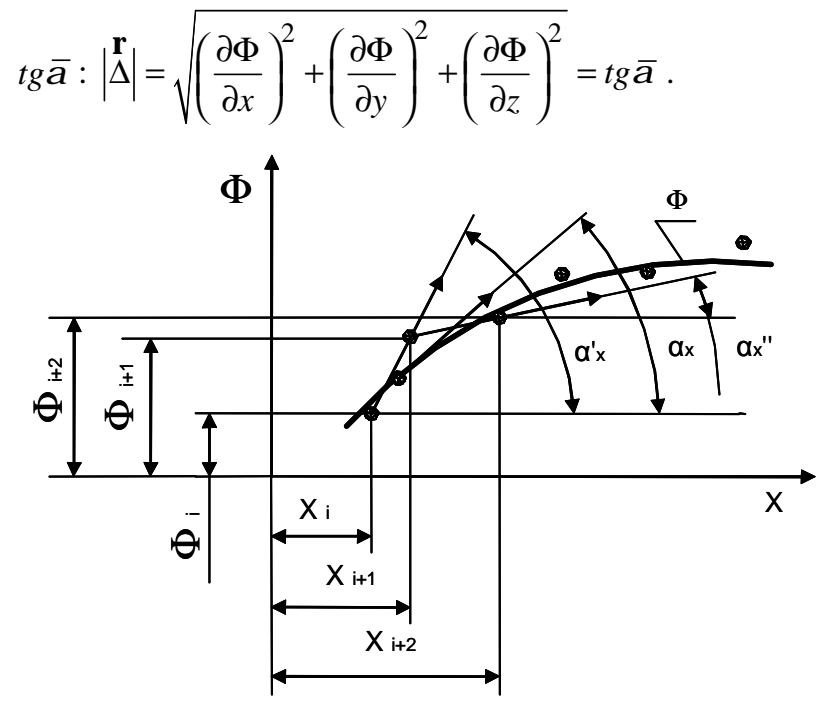

Fig 1. Graphical interpretation of measurement results

According to Fig 1, the value of $\operatorname{tg} \alpha_{x}^{\prime}$ can be approximately determined by taking the adjacent values of $\Delta \mathrm{x}$ :

$$
\operatorname{tg} \alpha_{x}^{\prime}=\frac{\Phi_{i+1}-\Phi x_{i}}{x_{i+1}-x_{i}}
$$

Although calculating the values of $\operatorname{tg} \alpha_{x}^{\prime \prime}$ according to the adjacent values $\Delta x_{i+2}$ and $\Delta x_{i+1}$, it would be

$$
\operatorname{tg} \alpha_{x}^{\prime \prime}=\frac{\Phi_{i+2}-\Phi_{i+1}}{x_{i+2}-x_{i+1}},
$$

and it will result in $\operatorname{tg} \alpha_{x}^{\prime \prime}<\operatorname{tg} \alpha_{x}^{\prime}$. It is apparent from Fig 1 that by calculating the values of $\operatorname{tg} \alpha_{x}$, random errors will have a big influence on the result.

They can appear in a different way during the repeated measurements of the surface. The methods must be applied that permit to define the features of the gradient on the surface when more accurate evaluation of variation of the systematic errors is needed. Using the method of mathematical regression, the law of variation of the systematic error in local intervals $x_{i}, x_{i+1}, \ldots, x_{i+n}$, $\mathrm{y}_{\mathrm{i}}, \mathrm{y}_{\mathrm{i}+1}, \ldots, \mathrm{y}_{\mathrm{i}+\mathrm{n}}$, and $\mathrm{z}_{\mathrm{i}}, \mathrm{z}_{\mathrm{i}+1}, \ldots, \mathrm{z}_{\mathrm{i}+\mathrm{n}}$ can be determined by polynomials

- for the volume:

$\Phi=a_{1}+a_{2} x+a_{3} y+a_{4} z+a_{5} x^{2}+a_{6} y^{2}+a_{7} z^{2}+a_{8} x^{3}+a_{9} y^{3}+a_{10} z^{3} ;$

- for the plane:

$$
\Phi=a_{1}+a_{2} x+a_{3} y+a_{4} x^{2}+a_{5} y^{2}+a_{6} x^{3}+a_{7} y^{3} ;
$$

- $\quad$ for coordinate measurements:

$\Phi=\mathrm{a}_{1}+\mathrm{a}_{2} \mathrm{x}+\mathrm{a}_{3} \mathrm{x}^{2}+\mathrm{a}_{4} \mathrm{x}^{3}$.

Here $a_{1}, a_{2}, \ldots a_{10}$ are the values of coefficients.

A least square method must be used for determination of the variable coefficients $a_{1}, a_{2}, \ldots, a_{n}$ : 


$$
A=\left(Y^{T} Y\right)^{-1} Y^{T} \bar{\Phi}
$$

Here A is a column of vector of the coefficients of variables $\mathrm{a}_{1}, \mathrm{a}_{2}, \ldots, \mathrm{a}_{\mathrm{n}} ; Y$ is a rectangular matrix created according to method described in paper 7 ; and $\Phi$ is a column of vector of the values of errors determined by experimental trials.

Analysis of the expressions (6), (7), and (8) show that each coordinate under the different powers has three additives and one free member $\mathrm{a}_{1}$. In this case, there will be 10; 7 and 4 unknown members in the polynomials in (6), (7) and (8). So, for determination of their values there is a need to construct the same number of equations. The more of equations that are created the more accurate the values of the coefficients $a_{1}, a_{2}, \ldots, a_{n}$ will be determined. Every equation will be created for a separate value of $\Phi_{\mathrm{i}}$ $(\mathrm{i}=1,2 \ldots)$. The number of equations necessary for this purpose can be calculated in such a way:

$$
u=n+b
$$

Here $n$ is the number of unknown coefficients of the polynomial; $b \geq 1$ is freely chosen number showing the number of equations that will be created (more than unknown coefficients). Then the points $r_{x}, r_{y}$, and $r_{z}$ for each coordinate will be calculated using this expression:

$$
\mathrm{r}_{\mathrm{x}}=\mathrm{r}_{\mathrm{y}}=\mathrm{r}_{\mathrm{z}}=\mathrm{r}=\mathrm{u} / \mathrm{p}
$$

Here $p$ is the different number of coordinates consisting in the calcukation.

In such a way, in the polynomial expressing a local surface, the number of coefficients is $n=10$ and $p=3$. It is better to select the value of the unknown coefficient $b$ in such a way that $r_{x}, r_{y}, r_{z}$ are integers. When choosing $b$ $=2$, it will be noted that $r_{x}=r_{y}=r_{z} \approx 4$. The same calculations could be performed in the cases (7) and (8).

By choosing a value for $b$, we need to know the number of points for each coordinate $(x, y$, and $z)$. The bigger the values are for $r_{x}, r_{y}$, and $r_{z}$, the more $\Phi_{\mathrm{i}}$ points will be used for the assessment of changes of errors at these points. This is $r_{x}=r_{y}=r_{z} \approx 4$ for the surface analyzed just before. For coordinate measurements expressed by (8), when $b=1, \quad n$ would be $u=n+b=4+1=5$, then $r_{x}=u / p=5 / 1=5$.

For the case discussed (Fig 1) five points must be used for assessment of the systematic errors. By drawing the diagram of the third order $\Phi$ (Fig 1), the closest approach to the law of variation of the systematic error would be the least square method. The values of partial derivatives calculated according to this diagram will serve to better determine the values of the local surface gradient and its constituents. This means that the method of analysis based on mathematical regression for determination of gradient or its constituents (proposed in papers [8, 7, and 3]) is more accurate than the method of calculation using the adjacent values of $\Phi_{\mathrm{i}}$ and $\Phi_{i+1}$. It must be noted that when using the latter method filtering separates a random part of the errors. This helps to explain the consistency and adequate accuracy of the modified surface discretization methods. This is the first main feature of its efficiency.
The second feature of efficiency of the modified surface discretization method shows the shortest wavelength of constituents of errors made in industry that can be evaluated by volume discretization.

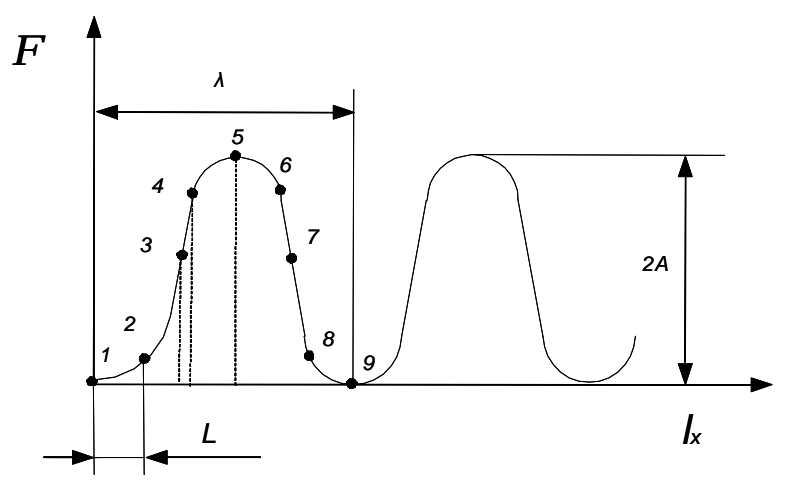

Fig 2. A simplified diagram of error distribution

In the simplified diagram of error distribution (Fig 2 ) the pitch of discretization of coordinate measurement is indicated by $L$ and by $\lambda-$ a wavelength of its constituent. The number of the pitches of discretization in the wavelength can be calculated by:

$$
s=\lambda / L \text {. }
$$

In case of $(n+b) p<\lambda /(2 L)$, for example, points 1 to 7 (Fig 2), is impossible to express the curve of error constituents by the polynomial of the third order. A significant part of the information is filtered off. In this case, the regression polynomials (6), (7), and (8) will adequately describe the law of distribution of the error constituents. Therefore, the expressions $(n+b) p<\lambda /(2 L)$ or $(n+b) p L<\lambda / 2$ will determine the second feature of the efficiency of the methods in use.

Now the ratio can be analyzed:

$$
\begin{aligned}
& w=s / r_{x}=\frac{\lambda / L}{u / p}=\frac{\lambda p}{u L}=\frac{\lambda p}{L(n+b)}=\frac{s p}{n+b} \\
& w=s / r_{y} \\
& w=s / r_{z}
\end{aligned}
$$

which shows a ratio between the wavelength, expressed by the number of pitches of discretization and the number of points falling to each coordinate.

By using less than half of wavelength $r^{\prime}$ for determination of unknown coefficients of the regression polynomial, according to (13) such expressions can be written:

$$
\begin{aligned}
& r^{\prime}=(s / 2)-m, w \leq \frac{s}{r^{\prime}} \leq \frac{2 \lambda}{\lambda-2 m L} \text { or } \\
& \frac{p}{L(n+b)} \leq \frac{2}{\lambda-2 m L} .
\end{aligned}
$$

Here $m$ is the integer showing at which number of discretization pitches the half of wavelength is shortened. 


\section{Analysis of the results}

The expression for determination of the shortest wavelength of constituents of the systematic errors and which will be efficient for use according to the method discussed is derived from (14):

$$
\lambda \geq 2\left(\frac{n+b}{p}+m\right) L,
$$

For example, by performing the measurements in the plane when $L=3 \mathrm{~mm}, n=7$ (expression (8)), $b=1$, and $p=2$ and $m=2$ for the plane measurement, then from (15) the value $\lambda \geq 36 \mathrm{~mm}$ will be received. In such a case, for all the constituents of systematic errors in the local surface that have wavelengths less than $36 \mathrm{~mm}$, the methods of modified discretization of measuring volume will act inefficiently. To improve its efficiency, when the length $\lambda$ doesn't comply with the requirements of length calculated by expression (15), the pitch of discretization must be decreased. It is evident from (15) that the dependence of $\lambda$ is linear to $L$. So, by decreasing the pitch of discretization $\mathrm{v}$ times, the wavelength of the pitch of measurement will remain in lessening at the same proportion.

The analysis performed showed that the effectiveness of the discretization methods (1) and (3) depends on the accuracy of evaluation of the parameters of the local surface errors and on the characteristics of the regression polynomial describing them. From expression (15), it is evident that the wavelength of $\lambda$ depends on the number of members of the regression polynomial. From one side, it seems that the more the number of members of the regression polynomial, the more accurately the errors of the local surface can be determined. This is not so however, since by expansion the number of members of the regression polynomial, the wavelength $\lambda$ of errors will be enlarged at the same time, and the errors inside the wavelength will be not evaluated. Decreasing of number of polynomial members will loose the accuracy of the description of the distribution of local surface errors. This is why the a priori information is needed about the surface to be measured before choosing the order of the polynomial.

\section{Conclusions}

After performing an analysis of the effectiveness of determining the pitch of discretization of the measurement surface by modified means, some conclusions can be made:

- The errors of the local surface and the characteristics of its gradient are more accurately determined by applying the methods of regression analysis. For this reason, the modified discretization methods that are discussed are valid and efficient;

- It is shown that the error constituents of short waves cannot be evaluated by using this modified surface discretization method. The dependence of the shortest waves of constituents of errors on the pitch of discretization of the surface and on the regression polynomial describing it is determined. The means to improve effectiveness of measurement are pointed out.

\section{References}

1. Bardell R., Balendran V., Sivayoganathan K. Accuracy analysis of 3D data collection and free-form modeling methods // Journal of Materials Processing Technology. - 2003. - No 33. - P. 26 - 33.

2. Cellary. A. Parameters selection in multipoint form measurement // XVI IMEKO World Congress "IMEKO 2000" proceedings. - Vienna: Austrian society for measurement and automation, 2000. - Vol. VIII. - P. 45 - 50.

3. Giniotis V., Mariūnas D. Differentially modified discretization method of dimensional space // Proceedings of the "The $2^{\text {nd }}$ International Symposium on Measurement, Analysis and Modeling of Human Function", "The $1^{\text {st }}$ Mediterranean Conference on Measurement", June 14 - 16, 2004 Genova, Italy. 2004. - P. 381 - 385.

4. Kim W.-S., Raman S.. On the selection of flatness measurement points in coordinate measuring machine inspection // International Journal of Machine Tools and Manufacture. Design Research and Application. 2000. - No 40. - P. 427 - 443.

5. Lee G., Mou J., Shen Y. Sampling strategy design for dimentional measurement of geometric features using coordinate measuring machine // International Journal of Machine Tools and Manufacture. - 1997. No 37(7). - P. 917 - 934.

6. Lin Z.C., Lin W.S. Measurement point prediction of flatness geometric tolerance by using grey theory // Journal of the International Societies for Precision Engineering and Nanotechnology. Precision Engineering. - 2001. - No 25. - P. 171 - 184.

7. Mariūnas D. Integrative modified discretization method of dimentional space // Proceeding of XIV International Symposium"Research, Practice and didactics in modern Machine Building", Stralsund, Germany, May 5 - 8, 2004. - 2004. - P. 61 - 68.

8. Mariūnas D. Modified of Measurement space discretization Method // Matavimai. - Kaunas, 2003. - Nr.2 (26). - P.36 - 40.

9. Summerhays K.D., Henke R.P., Baldwin J.M. et al. Optimizing discrete point sample patterns and measurement data analysis on internal cylindrical surfaces with systematic form deviations // Journal of the International Societies for Precision Engineering and Nanotechnology. Precession Engineering. - 2002. - No 26. - P. 105 - 121.

10. Woo T.C., Liang R., Hsieg C.C. et al. Efficient sampling for surface measurement // Journal of Manufacturing Systems. - 1995. -. No 14 (5). - P.345 $-354$.

11. Yau H.T., Menq C.H. Automate CMM path planning for dimensional inspection of dies and molds having complex surfaces // International Journal of Machine Tools and Manufacture. - 1995. - No 35 (6). - P. 861 -876 . 